\title{
VANTAGENS DE CITAÇÃO DO ACESSO ABERTO EM PERIÓDICOS SELECIONADOS DA CIÊNCIA DA INFORMAÇÃO: UMA ANÁLISE AMPLIADA AOS INDICADORES ALTMÉTRICOS
}

\author{
VENTAJAS DE CITA DEL ACCESO ABIERTO EN \\ REVISTAS SELECCIONADAS DE LA CIENCIA DE LA \\ INFORMACIÓN: UN ANÁLISIS EXTENDIDO A LOS \\ INDICADORES ALTMÉTRICOS
}

\author{
Paulo Roberto Cintra* \\ Ariadne Chloe Furnival** \\ Douglas Henrique Milanez**
}

\begin{abstract}
RESUMO
Introdução: $O$ acesso aberto diz respeito à literatura científica disponibilizada a qualquer usuário sem custos e livre das restrições de copyright e de licenciamento para seu reuso. Espera-se, de acordo com a hipótese do Open Access Citation Advantage, um aumento no número total de citações recebidas pelos artigos disponibilizados em acesso aberto com relação àqueles em acesso restrito. Objetivo: Analisar as possíveis vantagens de citações e menções na web social que o acesso aberto pode oferecer à área da Ciência da Informação. Metodologia: Foram analisados indicadores altmétricos e bibliométricos de citação de dois periódicos científicos: Journal of the American Society for Information Science e Scientometrics. A coleta dos dados foi realizada na Web of Science, Google Acadêmico, Altmetric.com e Mendeley. Resultados: Os resultados indicaram que o acesso aberto oferece vantagem quanto ao número de citações e de menções na web social recebidas pelos artigos em ambos as revistas. Verificou-se também que essa vantagem se mantém ao longo dos anos. Conclusões: A investigação confirma a hipótese do Open Access
\end{abstract}

*Doutorando do Programa de Pós-graduação em Política Científica e Tecnológica, da Universidade Estadual de Campinas. E-mail: paulocntr@yahoo.com

**Professora credenciada no programa de pós-graduação multidisciplinar, Ciência, Tecnologia e Sociedade (PPG-CTS) da UFSCar e do Programa de pós-graduação em Ciência da Informação (PPGCl). E-mail: chloe@ufscar.br

***Doutor em Ciência e Engenharia de Materiais pela Universidade Federal de São Carlos. Email: douglasmilanez@yahoo.com.br 
Paulo Roberto Cintra, Ariadne Chloe Furnival, Douglas Henrique Milanez.

Vantagens de citação do acesso aberto em periódicos selecionados da ciência da informação: uma análise ampliada aos indicadores altmétricos

Citation Advantage para os periódicos selecionados da área da Ciência da Informação e demonstra que essa vantagem se estende aos dados altmétricos.

Palavras-chave: Acesso Aberto. Periódicos. Ciência da Informação. Bibliometria. Altmetria.

\section{INTRODUÇÃO}

A Iniciativa Budapeste para o Acesso Aberto (Budapest Open Access Initiative - BOAI), realizada em Budapeste, na Hungria, pela Open Society Foundations (OSF), em dezembro de 2001, apontou que a Internet e a boa vontade dos cientistas em publicarem os resultados de suas pesquisas tornaram possível a origem do acesso aberto. Neste mesmo evento, definiu-se o acesso aberto como:

A literatura que é disponibilizada livremente na Internet pública, permitindo a qualquer usuário ler, baixar, copiar, distribuir, imprimir, pesquisar, ou manter links para os textos completos dos artigos, rastreá-los para indexação, passá-los como dados para software, ou usá-los para qualquer outro propósito legal, sem a existência de barreiras financeiras, legais ou técnicas que não sejam aquelas ligadas ao próprio acesso da internet. $A$ única limitação sobre a reprodução e distribuição, e o único papel dos direitos autorais nesse domínio, deve ser o controle do autor sobre a integridade de seu trabalho e o direito de ser devidamente reconhecido e citado (BOAl, 2016, tradução nossa).

A recomendação trazida pela Iniciativa Budapeste foi das duas estratégias basilares para o acesso aberto: i) através do autoarquivamento de documentos (green road - "via verde") e ii) pela criação de periódicos em acesso aberto (gold road - "via dourada"). Não obstante essas duas estratégias, a literatura ainda aponta a existência de um modelo híbrido que pode ser utilizado pelas revistas científicas de acesso restrito, no qual é oferecido aos autores a opção do pagamento de uma taxa de processamento de artigos (Article Processing Charges - APCs) para publicação dos seus artigos em acesso aberto imediatamente. De acordo com Pinfield et al. (2015), nestes casos, é preciso atentar para o risco de dupla-cobrança (doubledipping), uma vez que podem existir situações nas quais as editoras lucrem 
duas vezes, pelo mesmo conteúdo: $1^{\mathrm{a}}$ ) através da obtenção de receitas pelo recebimento dos APCs daqueles autores que queiram, ou que são obrigados pelos seus órgãos de fomento, a publicarem de imediato seus artigos em acesso aberto; e $2^{\mathrm{a}}$ ) pela manutenção da taxa de subscrição dos periódicos às instituições onde estes pesquisadores atuam, a fim de que tenham acesso ao conteúdo completo das revistas científicas.

Sublinha-se ainda que cada periódico científico pode autorizar o arquivamento de diferentes versões dos trabalhos a ela submetidos. Ou seja, dependendo das políticas de autoarquivamento do periódico, os autores poderão efetuar o arquivamento apenas do pre-print, do post-print ou mesmo da versão final dos artigos. Ademais, existem situações em que é exigido ainda um período de carência (embargo) entre a data de publicação do artigo na revista e o depósito desse documento nos repositórios institucionais ou sites pessoais.

As várias opções existentes para a publicação em acesso aberto explicam o relativo sucesso deste modelo de publicação científico. O relatório Proportion of open access peer-reviewed papers at the European and world levels - 2004-2011 (ARCHAMBAULT et al., 2013) publicado pelo European Commission DG Research \& Innovation, afirma que o Brasil, a Suiça, a Holanda e os Estados Unidos, já alcançaram seu ponto de inflexão (tipping point), ou seja, mais de $50 \%$ do número de artigos publicados pelo país são disponibilizados em acesso aberto. De acordo com Costa e Leite (2016), especificamente no caso do Brasil e de outros países da América Latina, este sucesso se deve à quantidade de revistas científicas em acesso aberto publicadas por editoras não comerciais e de associações científicas. Salientase que, segundo os autores, em 2013, o Brasil foi o segundo país com o maior número de periódicos em acesso aberto no Directory of Open Access Journals (DOAJ).

A adoção do modelo de acesso aberto, por ambas as vias "dourada" e "verde", historicamente, se deve a certos fatores negativos. Podem ser mencionados: i) os custos, acima das taxas de inflação, das assinaturas aos periódicos científicos; e ii) a concentração em um pequeno grupo de editoras 
Paulo Roberto Cintra, Ariadne Chloe Furnival, Douglas Henrique Milanez.

Vantagens de citação do acesso aberto em periódicos selecionados da ciência da informação: uma análise ampliada aos indicadores altmétricos

comerciais multinacionais, que, ao mesmo tempo, dependem do trabalho gratuito dos pares científicos para manter os altos padrões de qualidade do peer review. Cresce, assim, a consciência na comunidade científica da desigualdade de acesso à literatura científica pelo mundo que desproporcionalmente beneficia os países centrais e, concomitantemente, aumenta a propagação e aceitação dos modelos de "ciência aberta", arraigados no princípio de que o conhecimento científico é, acima de tudo, um bem público comum para o desenvolvimento e progresso econômico e social (STIGLITZ, 1999). O bem público, à luz do conceito advindo da Economia, possui como propriedades intrínsecas a não-rivalidade e a não-exclusividade. Ou seja, se um indivíduo, por exemplo, utilizar determinado teorema matemático, não haverá redução na quantidade disponível desse conhecimento para as demais pessoas da sociedade (não-rivalidade). Além disso, não é possível impedir que alguém usufrua desta informação (nãoexclusividade) (ROSEN; GAYER, 2014).

No que diz respeito ao desenvolvimento e progresso científico, Meadows (1999) e Targino (2000) consideram que a transmissão dos conhecimentos entre os pares é fundamental para tais fins, pois é através dessa comunicação que os resultados alcançados por um pesquisador podem ser testados, avaliados, certificados e aprovados pela comunidade acadêmica. Nesse sentido, a disponibilidade da produção científica tem um papel crucial em garantir que certos resultados da pesquisa científica suscitarão um impacto passível de gerar novos conhecimentos científicos. Na Bibliometria, costuma-se aferir o potencial impacto de uma dada pesquisa científica pelo número de citações que um dos produtos dessa pesquisa - o artigo científico - gera, entre outras medidas englobadas pelos estudos métricos. Dado esta medida de impacto, desde a incepção do movimento de acesso aberto, surgiu a pergunta se artigos amplamente acessíveis e, portanto, citáveis - seja pela via dourada, seja pela verde - seriam mais citados e, assim, potencialmente suscitariam maior impacto no avanço científico de um dado campo. Esta é uma hipótese que tem sido muito discutida na academia, tendo até recebido a sigla OACA, cujo significado é Open Access Citation Advantage. 
Paulo Roberto Cintra, Ariadne Chloe Furnival, Douglas Henrique Milanez.

Vantagens de citação do acesso aberto em periódicos selecionados da ciência da informação: uma análise ampliada aos indicadores altmétricos

Em comunicação seminal da área, Lawrence (2001) procurou estabelecer se, para o campo da Computação, existia tal vantagem de citação de artigos. Por se tratar de uma época anterior à consolidação do acesso aberto, o autor denominou essas publicações de "feely-available online articles". Os resultados levantados por Lawrence (2001, tradução nossa) demonstraram que existe uma vantagem para artigos disponíveis online: "O número médio de citações para artigos offline é 2.74 , e o número médio de citações para artigos online é 7.03, um aumento de 157\%". Estes resultados abriram o caminho para uma pauta de pesquisa prolífica para comprovar ou refutar a hipótese da OACA.

Conforme mostra Droescher e Silva (2015), diversos estudos já foram realizados com o intuito de investigar a existência da vantagem de citações para os mais variados campos do saber. Podem ser citadas pesquisas na área das Engenharias (ANTELMAN, 2004), nas Ciências Naturais (HARNAD; BRODY, 2004) e nas Ciências Sociais e Humanas (ATCHINSON; BULL, 2015). No relatório produzido por Archambault et al.(2013), por exemplo, os autores constataram a OACA para 22 campos do conhecimento. Entre os resultados encontrados, verificaram que, na média, todas as áreas apresentam alguma vantagem, ainda que para determinados casos o valor tenha sido pouco significante. Em um estudo mais recente de Archambault et al. (2016), os autores analisam a média de citações relativa para 3,3 milhões de artigos indexados na Web of Science, cuja publicação ocorreu entre 2007 e 2009. Como conclusão, os pesquisadores também confirmaram a hipótese do OACA para este grande conjunto de dados e mostraram que a vantagem é ainda maior para as publicações disponibilizadas em acesso aberto através da via verde. Swan (2010), entretanto, ressalta que também existem pesquisas que mostraram não existir nenhuma vantagem na quantidade de citações para determinados campos científicos, como na área da Economia (FRANDSEN, 2009).

Harnad e Brody (2004) e Harnad (2005) discutem os motivos pelos quais possa existir essa vantagem de citações do acesso aberto. Para os autores, ter acesso ao conteúdo científico produzido é importante e necessário para que a 
Paulo Roberto Cintra, Ariadne Chloe Furnival, Douglas Henrique Milanez.

Vantagens de citação do acesso aberto em periódicos selecionados da ciência da informação: uma análise ampliada aos indicadores altmétricos

citação aconteça, mas fazem a ressalva de que, sozinho, esse acesso não é condição suficiente para que a referência ocorra. Destarte, entre as razões que explicam a vantagem, podem ser citados o viés de qualidade e a vantagem de antecipação. Ou seja, os pesquisadores fazem uma seleção de seus próprios artigos e disponibilizam em acesso aberto apenas aqueles que consideram como sendo de maior qualidade. Além disso, os trabalhos cujos resultados são depositados em acesso aberto desde o estágio do pre-print possibilita que as citações ocorrem antecipadamente.

Não obstante esses estudos, Kousha e Thelwall (2007) discutem que atividade científica vai além da publicação de artigos em periódicos e que referências feitas tanto nos meios de comunicação formais, quanto em meios informais podem não ser detectados pelas atuais técnicas de análise de citação e indicadores bibliométricos tradicionais. Haustein et al. (2013) corroboram essa visão e afirmam que a avaliação do impacto de um artigo científico deve ir além da análise de citações, pois os pesquisadores nem sempre fazem a citação de todos os artigos que utilizaram durante a realização de suas pesquisas, ainda que estes trabalhos os tenham influenciado. Além disso, Kousha e Thelwall (2007) ressaltam que a Internet hoje permite a recuperação de parte dessa comunicação até então ignorada, sendo possível ampliar a verificação do impacto de uma pesquisa científica para além dos meios formais, através da análise das menções em mídias sociais e nos dados fornecidos pelos gerenciadores de referências.

Diante disto surge a Altmetria, que de acordo com Souza (2014), é a tradução do termo em inglês altmetrics, uma contração da expressão alternative metrics. A autora explica que esse subcampo das pesquisas métricas busca elaborar e utilizar indicadores a partir da quantificação estatística de dados coletados na web social, como o número de downloads de um artigo, a quantidade de compartilhamentos em redes sociais e as citações desses documentos em blogs e sites de notícias. De acordo com Galligan e Dyas-Correia (2013), a criação da Altmetria só foi possível devido ao aumento do número de usuários na Internet e também do aumento da interação entre eles, sobretudo, em redes sociais. Nesse sentido, aqueles que eram apenas 
Paulo Roberto Cintra, Ariadne Chloe Furnival, Douglas Henrique Milanez.

Vantagens de citação do acesso aberto em periódicos selecionados da ciência da informação: uma análise ampliada aos indicadores altmétricos

visitantes de páginas, passaram também a dialogar e estabelecer uma comunicação digital integrada à estrutura da comunidade científica. Assim, os rastros (trails) das pegadas digitais deixadas pelos usuários na web podem ser registrados instantaneamente e transformadas em informações passíveis de serem lidas e analisadas.

Priem et al. (2010) acreditam ainda que a Altmetria pode contribuir como medida complementar às tradicionais métricas, como a bibliometria. Nesse sentido, para Wang et al. (2015), os dados altmétricos também podem ser utilizados como um indicador suplementar à investigação de OACA. Os autores, por exemplo, propuseram-se a comparar os artigos da revista Nature Communications em acesso aberto, com aqueles em acesso restrito, analisando a quantidade de citações recebidas, o número de visualizações dos artigos e a discussão obtida nas redes sociais Twitter e Facebook. Como resultado, confirmaram a hipótese de vantagem de citação para os artigos em acesso aberto e averiguaram que essa vantagem também se estendia aos dados altmétricos. Assim, os artigos em acesso aberto tendem a receber mais atenção na web social do que aqueles que não eram disponibilizados da mesma forma.

Para o caso específico do campo da Ciência da Informação e Biblioteconomia, Xia et al. (2011) realizaram uma investigação sobre o OACA em 20 periódicos da área. Como resultado, os autores encontraram que há uma correlação positiva e estatisticamente significativa entre o número de citações e a disponibilidade dos artigos em acesso aberto. Além disso, os pesquisadores constataram ainda que o número de citações também aumenta quando a quantidade de cópias em acesso aberto disponibilizadas na web cresce. Para Xia et al. (2011), essa situação acontece quando um mesmo artigo é depositado online por outras pessoas além dos autores, como os bibliotecários e os alunos assistentes.

Outro estudo relevante é o de Maflahi e Thelwall (2016), pois traz uma análise da relação entre o número de citações em quatro periódicos da área da Ciência da Informação, indexados na base de dados Scopus, e a quantidade de "leitores" (readers) desses artigos no Mendeley, um gestor de referências da 
empresa Elsevier, considerado como uma importante fonte de informação para obtenção de dados altmétricos. Nesse sentido, os autores verificaram que existe uma correlação positiva entre as duas variáveis, indicando que a quantidade de leitores do Mendeley pode refletir no número de citações obtidas pelos artigos. Relação que se mantêm tanto para artigos mais novos, quanto para os mais antigos.

Constata-se, portanto, que ainda há uma incipiência de estudos que avaliem concomitantemente a OACA e as menções desses artigos na web social. Além disso, poucas pesquisas têm verificado se a vantagem é decorrente do pagamento das taxas de processamento de artigos ou do depósito dessas publicações em repositórios institucionais (via verde). Portanto, a partir dessas acepções, destaca-se que o objetivo deste estudo foi investigar as possíveis vantagens que o acesso aberto pode oferecer à área da Ciência da Informação, avaliando se a livre disponibilidade dos artigos à comunidade científica, através de APCs e autoarquivamento, favorece um aumento no número de vezes que uma publicação é citada ou mencionada na web social.

\section{PROCEDIMENTOS METODOLÓGICOS}

\subsection{Seleção dos periódicos na área de $\mathrm{Cl}$}

A seleção de revistas científicas considerou a lista de 85 periódicos fornecida pelo Journal Citation Reports (JCR), publicação da Thomson Reuters, edição Social Science, de 2014, na categoria Information Science \& Library Science. Essa escolha foi motivada pelo fato do JCR estar vinculada a Web of Science, base bibliográfica reconhecida pela indexação de revistas científicas de grande relevância no meio acadêmico e pelo fato da base ser recorrentemente utilizada em estudos bibliométricos (OKUBO, 1997). A partir desta listagem, realizou-se uma caracterização das políticas desses periódicos acerca da disponibilização de artigos em acesso aberto através do sistema SHERPA/RoMEO, pelo qual foi possível estabelecer três grupos: Acesso Aberto (15 periódicos), Acesso Fechado (5 periódicos) e Híbridos (65 periódicos). 
Não foram encontrados meios gratuitos para se automatizar a coleta dos dados altmétricos, que ficaram sujeitos à verificação manual. Dessa forma, houve a necessidade de se estabelecer filtros que permitissem a seleção de um número viável de publicações para a realização desse estudo. Assim, o primeiro recorte estabelecido foi através da seleção dos periódicos classificados como híbridos, ou seja, 65 revistas científicas. Destas, foram tomadas aquelas que apresentaram classificação estratificada dentro das Áreas de Avaliação Interdisciplinar e de Ciências Sociais Aplicadas I estabelecidas pelo WebQualis/CAPES (atual Plataforma Sucupira). Por fim, foram escolhidas as duas revistas com maior fator de impacto no momento da seleção: Scientometrics (2.183) e Journal of the American Society for Information Science and Technology (1.846).

\subsection{Coleta de dados e elaboração dos indicadores}

A coleta dos metadados das publicações desses dois periódicos foi realizada através da base de dados bibliográfica Web of Science, em sua Coleção Principal, no dia 22 de outubro de 2015. Foi estabelecido um recorte temporal, de janeiro de 2007 a dezembro de 2014, e a opção exclusiva de artigos de pesquisa. A escolha do período se deve à necessidade de tempo para que uma citação realizada possa ser devidamente indexada e, posteriormente, computada pela base de dados. Cabe salientar que o periódico Journal of the American Society for Information Science and Technology (JASIST) foi descontinuado no ano de 2013 , com a substituição de seu nome e número de ISSN. Nesse sentido, para esta revista considerou-se apenas os dados referentes até 2013. Destaca-se ainda que a Web of Science não indica se um artigo está disponibilizado em acesso aberto, por isso, essa informação foi obtida pelo acesso ao site de cada revista e a realização de verificação individual, dentro do período estabelecido, de quais publicações estavam disponibilizados em acesso aberto.

Outrossim, conforme indicado por Antelman (2004), a fim verificar a disponibilidade dos artigos pela via verde, buscou-se através do Google Acadêmico o depósito dos artigos em repositórios digitais, sites pessoais dos autores ou em outras fontes nas quais fosse possível encontrar o texto 
Paulo Roberto Cintra, Ariadne Chloe Furnival, Douglas Henrique Milanez.

Vantagens de citação do acesso aberto em periódicos selecionados da ciência da informação: uma análise ampliada aos indicadores altmétricos

completo dessas publicações sem barreiras ao acesso. Esta pesquisa foi realizada em ambiente externo ao da instituição de ensino superior, a fim de garantir que o documento encontrado realmente esteja em acesso aberto e não tenha sido disponibilizado pelo portal do Periódico CAPES. Além da verificação de disponibilidade do artigo em acesso aberto, buscou-se averiguar em qual tipo de site esse documento estava localizado e se a versão arquivada era preprint ou post-print. Ainda que existissem cópias do artigo em mais de um site, para a análise, considerou-se apenas o primeiro resultado retornado pelo mecanismo de busca. Além disso, segundo Willinsky (2006), um modo alternativo à criação e manutenção de repositórios pelas instituições, é a disponibilização de páginas pessoais (homepages) a seus pesquisadores, dentro do site da própria universidade ou centro de pesquisa. Por esse motivo, tanto os repositórios institucionais, quanto as homepages universitárias, foram considerados como pertencentes à nomenclatura "Site Institucional".

A respeito dos dados altmétricos, não existe na literatura um consenso sobre qual o melhor procedimento para se aferir essas informações. Por esse motivo, foram coletados dados do Altmetric.com e do Mendeley. Nesse sentido, para cada artigo, foi aplicado o Altmetric Bookmarklet, aplicativo gratuito disponibilizado pelo site Altmetric.com. Para os dados do Mendeley, foram verificados o número de leitores (readers), que fizeram o download das referências dos artigos em análise. Salienta-se que a coleta dos dados altmétricos foi realizada durante o mês de janeiro de 2016.

Por fim, para verificação da hipótese de OACA, foram comparadas as médias de citação dos artigos e das menções na web social, para os artigos disponibilizados em acesso aberto e em acesso restrito. Destaca-se que foram avaliados, separadamente, o acesso aberto oferecido através do pagamento dos APCs e pelo autoarquivamento dos documentos em repositórios institucionais abertos.

\section{RESULTADOS E DISCUSSÃO}

Considerando o total de publicações científicas em ambos periódicos no período selecionado, verificou-se que o percentual de artigos que estão 
Paulo Roberto Cintra, Ariadne Chloe Furnival, Douglas Henrique Milanez.

Vantagens de citação do acesso aberto em periódicos selecionados da ciência da informação: uma análise ampliada aos indicadores altmétricos

disponíveis em acesso aberto corresponde à 4,0\% para JASIST e 4,1\% para Scientometrics. O baixo percentual pode estar associado ao custo para processamento dos artigos para serem publicados em acesso aberto, que é o mesmo em ambos os periódicos. Apesar do valor relativamente baixo e do período de embargo para que o autor possa disponibilizar a publicação, por exemplo, em repositórios institucionais, mais da metade das publicações em ambos os periódicos podem ser encontradas em seu texto completo e sem barreiras ao acesso.

Tabela 1. Caracterização dos periódicos selecionados quanto a critérios relacionados ao acesso aberto

\begin{tabular}{l|c|c}
\hline Periódico & JASIST & Scientometrics \\
\hline $\begin{array}{l}\text { Período de cobertura da } \\
\text { pesquisa }\end{array}$ & Jan. 2007 - Dez. 2013 & Jan. 2007 - Dez. 2014 \\
\hline $\mathrm{N}^{\circ}$ de artigos publicados & 1269 & 1721 \\
\hline Valor APC (US\$) & 3000 & 3000 \\
\hline Período de embargo & 24 & 12 \\
\hline $\begin{array}{l}\mathrm{N}^{\circ} \text { de artigos em Acesso Aberto } \\
\text { no Periódico (APC) }\end{array}$ & 51 & 867 \\
\hline $\begin{array}{l}\mathrm{N}^{\circ} \text { total de artigos em Acesso } \\
\text { Aberto (APC + via verde) }\end{array}$ & 814 & $50,4 \%$ \\
\hline $\begin{array}{l}\text { \% Acesso Aberto Total (APC + } \\
\text { via verde) }\end{array}$ & $64,1 \%$ & 71 \\
\hline
\end{tabular}

Fonte: Dados da pesquisa elaborados da Web of Science, Google Acadêmico e site dos periódicos.

O Gráfico 1 apresenta os locais onde foram encontradas as publicações em acesso aberto - seja pelo pagamento de APC ou após o período de embargo - de acordo com a versão arquivada (pre-print ou post-print). Os autores mostraram preferência pelo autoarquivamento em sites institucionais, na proporção de $44,8 \%$ para JASIST e $41,6 \%$ no Scientometrics. A rede social ResearchGate foi o segundo local mais utilizado para o depósito dessas publicações, em ambos os periódicos, com um total de 260 artigos do JASIST e de 322 documentos do Scientometrics. O ResearchGate é uma plataforma online, cujo objetivo é conectar e contribuir para formação de redes de colaboração entre os pesquisadores, de modo que o compartilhamento e 
Paulo Roberto Cintra, Ariadne Chloe Furnival, Douglas Henrique Milanez.

Vantagens de citação do acesso aberto em periódicos selecionados da ciência da informação: uma análise ampliada aos indicadores altmétricos

acesso ao conhecimento científico produzido seja facilitado (RESEARCHGATE, 2016). Quanto ao autoarquivamento, aferiu-se uma predileção às versões post-print das publicações, na razão de 53,8\% para JASIST e $61,6 \%$ no Scientometrics.

Gráfico 1. Locais utilizados para arquivamento dos artigos

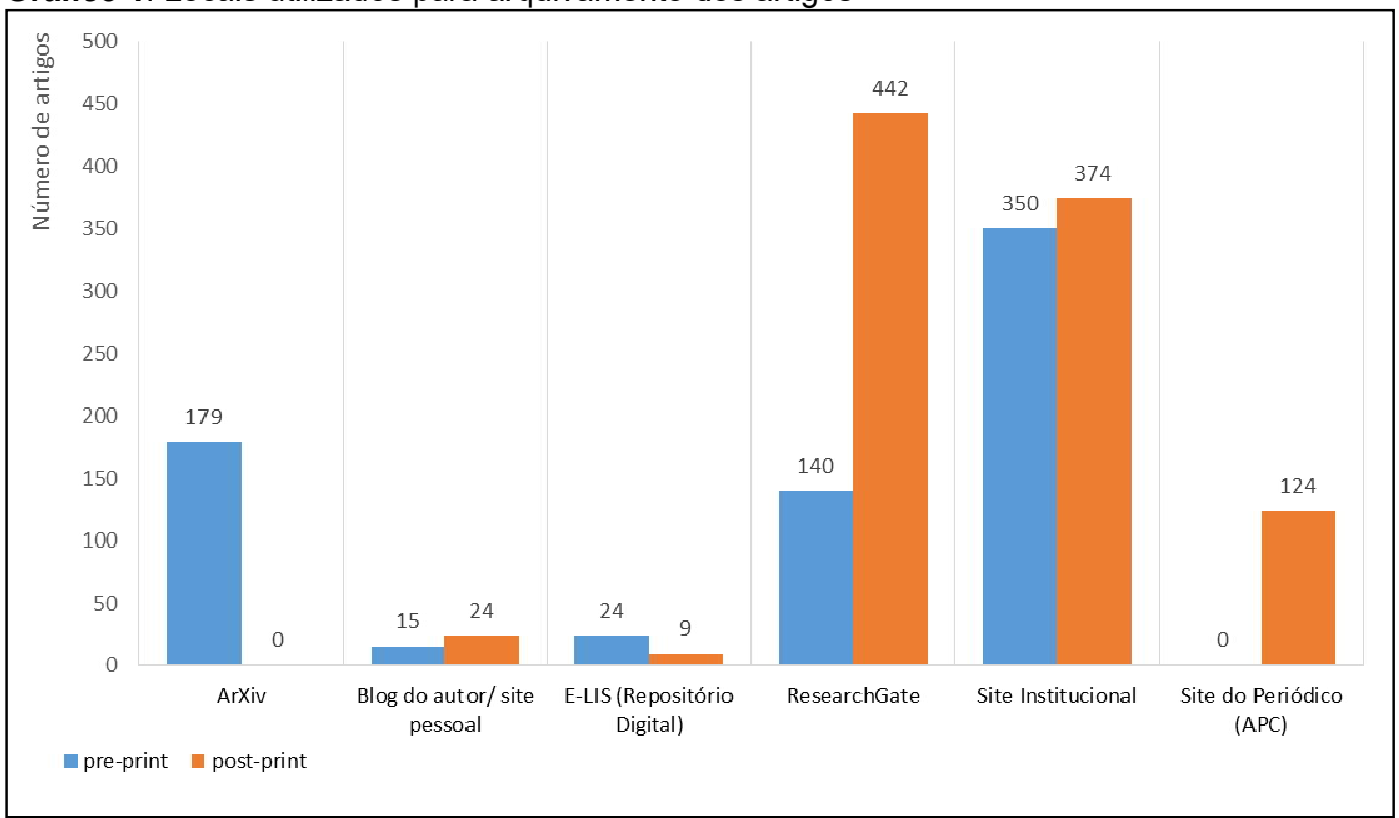

Fonte: Dados da pesquisa.

O acesso aberto oferece uma vantagem no número de citações para ambos os periódicos, independente do ano analisado, conforme Tabelas 2 e 3. O percentual médio referente a essa vantagem foi de $225,2 \%$ para o caso do JASIST e de $177 \%$ para o Scientometrics. Além disso, no que concerne ao Journal of the American Society for Information Science and Technology, existe uma maior vantagem para os artigos disponibilizados através do autoarquivamento em repositórios abertos na web, pois eles tendem a receber, em média, mais citações do que aqueles disponibilizados via pagamento de APC. Por outro lado, para o periódico Scientometrics, nos cinco primeiros anos após a publicação, os artigos com maior vantagem foram aqueles cujos autores arcaram com os custos das taxas de processamento. 
Paulo Roberto Cintra, Ariadne Chloe Furnival, Douglas Henrique Milanez.

Vantagens de citação do acesso aberto em periódicos selecionados da ciência da informação: uma análise ampliada aos indicadores altmétricos

Tabela 2. Média das citações para artigos, em acesso fechado e acesso aberto, do periódico

\begin{tabular}{|c|c|c|c|c|c|c|c|}
\hline & \multirow[b]{2}{*}{$\begin{array}{l}\text { Acesso } \\
\text { Fechado }\end{array}$} & \multicolumn{6}{|c|}{ Acesso Aberto } \\
\hline & & APC & $\begin{array}{c}\text { APC } \\
\text { Acesso } \\
\text { Fechado }\end{array}$ & $\begin{array}{c}\text { Via } \\
\text { Verde }\end{array}$ & $\begin{array}{l}\frac{\text { Via Verde }}{\text { Acesso }} \\
\text { Fechado }\end{array}$ & $\begin{array}{c}\text { Total AA } \\
\text { (APC + via } \\
\text { verde) }\end{array}$ & $\begin{array}{l}\text { Total AA } \\
\text { Acesso } \\
\text { Fechado }\end{array}$ \\
\hline $\begin{array}{c}1^{\circ} \\
\text { ano }\end{array}$ & 0,24 & 0,39 & $162,5 \%$ & 0,45 & $188,0 \%$ & 0,45 & $186,3 \%$ \\
\hline $\begin{array}{c}2^{\circ} \\
\text { ano }\end{array}$ & 1,00 & 1,41 & $141,3 \%$ & 2,22 & $222,0 \%$ & 2,17 & $217,1 \%$ \\
\hline $\begin{array}{l}3^{\circ} \\
\text { ano }\end{array}$ & 1,54 & 2,29 & $149,0 \%$ & 3,03 & $196,9 \%$ & 2,99 & $194,1 \%$ \\
\hline $\begin{array}{c}4^{\circ} \\
\text { ano }\end{array}$ & 1,66 & 1,66 & $99,8 \%$ & 3,23 & $194,6 \%$ & 3,14 & $189,2 \%$ \\
\hline $\begin{array}{c}5^{\circ} \\
\text { ano }\end{array}$ & 1,52 & 1,84 & $120,5 \%$ & 3,06 & $200,4 \%$ & 2,98 & $195,6 \%$ \\
\hline $\begin{array}{c}6^{\circ} \\
\text { ano }\end{array}$ & 1,49 & 2,08 & $140,1 \%$ & 3,18 & $213,7 \%$ & 3,12 & $209,3 \%$ \\
\hline $\begin{array}{c}7^{\circ} \\
\text { ano }\end{array}$ & 1,15 & 2,35 & $204,2 \%$ & 3,17 & $275,5 \%$ & 3,12 & $271,6 \%$ \\
\hline $\begin{array}{c}8^{\circ} \\
\text { ano }\end{array}$ & 1,08 & 2,06 & $190,0 \%$ & 2,75 & $253,9 \%$ & 2,71 & $250,4 \%$ \\
\hline $\begin{array}{c}9^{\circ} \\
\text { ano }\end{array}$ & 1,03 & 1,29 & $124,4 \%$ & 3,36 & $324,9 \%$ & 3,24 & $313,1 \%$ \\
\hline
\end{tabular}

Fonte: Web of Science, Google Acadêmico e site dos periódicos.

Nota: "Acesso Fechado" foi utilizado para os artigos com acesso restrito nas revistas e que não possuíam nenhuma versão em acesso aberto na web.

Tabela 3. Média das citações para artigos, em acesso fechado e acesso aberto, do periódico Scientometrics

\begin{tabular}{|c|c|c|c|c|c|c|c|}
\hline \multirow{2}{*}{\multicolumn{2}{|c|}{$\begin{array}{c}\text { Acesso } \\
\text { Fechado }\end{array}$}} & \multicolumn{6}{|c|}{ Acesso Aberto } \\
\hline & & APC & $\begin{array}{c}\text { APC } \\
\text { Acesso } \\
\text { Fechado }\end{array}$ & $\begin{array}{c}\text { Via } \\
\text { Verde }\end{array}$ & $\begin{array}{c}\text { Via Verde } \\
\text { Acesso } \\
\text { Fechado }\end{array}$ & $\begin{array}{c}\text { Total AA } \\
\text { (APC + via } \\
\text { verde) }\end{array}$ & $\begin{array}{l}\text { Total AA } \\
\text { Acesso } \\
\text { Fechado }\end{array}$ \\
\hline $\begin{array}{c}1^{\circ} \\
\text { ano }\end{array}$ & 0,30 & 0,54 & $182,4 \%$ & 0,47 & $158,3 \%$ & 0,47 & $159,6 \%$ \\
\hline $\begin{array}{c}2^{\circ} \\
\text { ano }\end{array}$ & 1,44 & 2,48 & $172,4 \%$ & 2,03 & $141,2 \%$ & 2,09 & $145,6 \%$ \\
\hline $\begin{array}{c}3^{\circ} \\
\text { ano }\end{array}$ & 1,82 & 4,52 & $248,6 \%$ & 2,80 & $153,9 \%$ & 2,84 & $156,3 \%$ \\
\hline $\begin{array}{c}4^{\circ} \\
\text { ano }\end{array}$ & 1,87 & 4,34 & $231,5 \%$ & 2,95 & $157,6 \%$ & 3,02 & $161,1 \%$ \\
\hline $\begin{array}{c}5^{\circ} \\
\text { ano }\end{array}$ & 1,89 & 4,76 & $251,8 \%$ & 2,98 & $157,9 \%$ & 3,13 & $165,4 \%$ \\
\hline $\begin{array}{c}6^{\circ} \\
\text { ano }\end{array}$ & 1,54 & 3,83 & $249,7 \%$ & 2,92 & $190,1 \%$ & 3,02 & $197,0 \%$ \\
\hline $\begin{array}{r}7^{\circ} \\
\text { ano }\end{array}$ & 1,26 & 1,92 & $152,7 \%$ & 2,92 & $232,5 \%$ & 2,85 & $227,0 \%$ \\
\hline $\begin{array}{c}8^{\circ} \\
\text { ano } \\
\end{array}$ & 1,63 & 2,06 & $126,6 \%$ & 2,96 & $181,8 \%$ & 2,89 & $177,6 \%$ \\
\hline $\begin{array}{r}9^{\circ} \\
\text { ano }\end{array}$ & 1,22 & 2,25 & $183,8 \%$ & 2,50 & $204,3 \%$ & 2,47 & $201,6 \%$ \\
\hline
\end{tabular}

Fonte: Web of Science, Google Acadêmico e site dos periódicos

Nota: "Acesso Fechado" foi utilizado para os artigos com acesso restrito nas revistas e que não possuíam nenhuma versão em acesso aberto na web. 
Com base nas informações fornecidas pelo Gráfico 2, é possível atestar que os dados altmétricos e de citação do Google Acadêmico, de modo geral, corroboram para confirmação de OACA em periódicos da Ciência da Informação. $O$ acesso aberto proporcionado através do pagamento das taxas de processamento de artigos oferece - embora o valor seja ligeiramente superior - uma maior vantagem no número de menções na web social (Mendeley e dados do Altmetric.com), enquanto que o autoarquivamento dos artigos pelos autores tende a elevar a quantidade de citações que esses trabalhos recebem.

Gráfico 2. Análise de OACA em diferentes fontes de informação

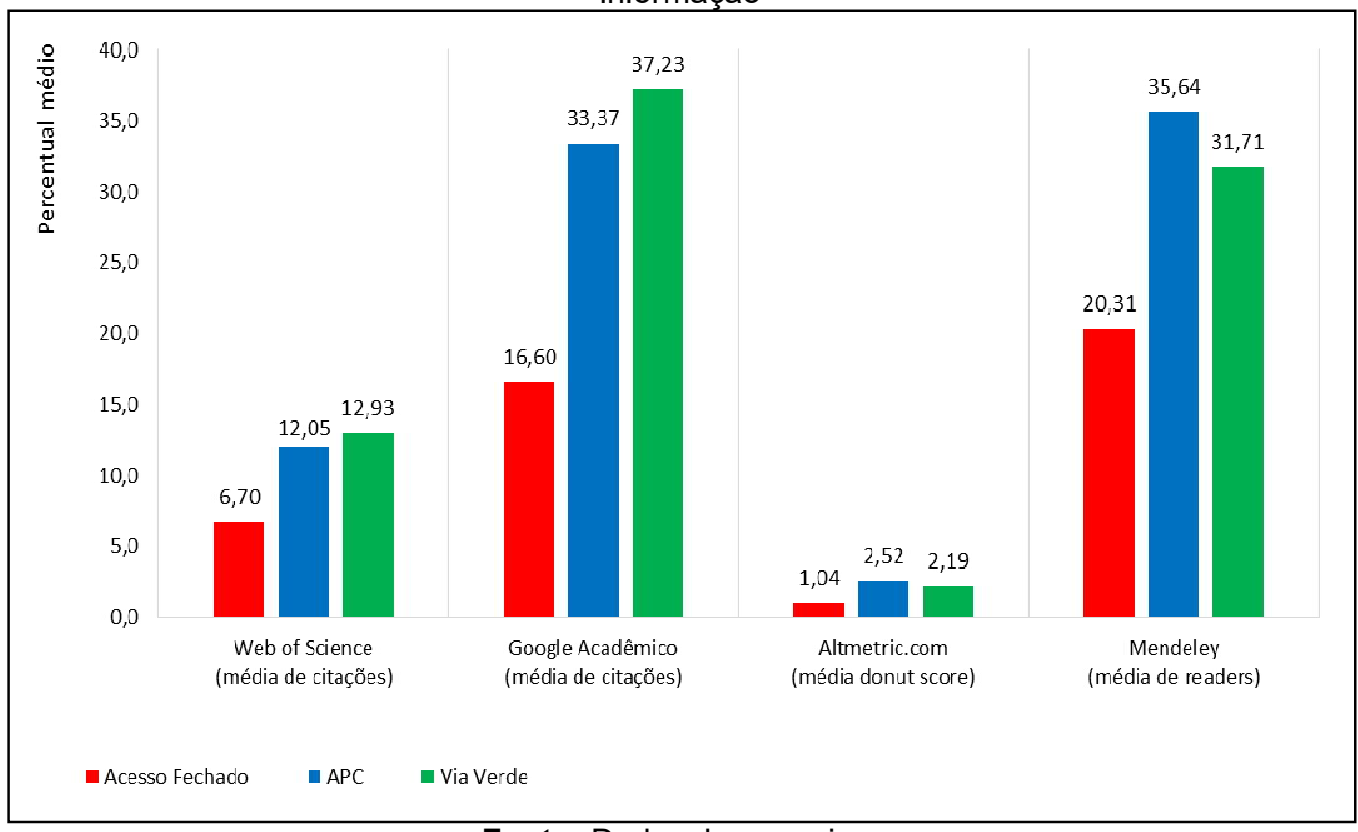

Fonte: Dados da pesquisa.

\section{CONCLUSÃO}

A investigação permitiu inferir que o arquivamento de um artigo em acesso aberto, seja pela via verde ou através do pagamento de APCs, possibilita, de fato, uma vantagem no número potencial de citações e de menções na web social que as publicações da área da Ciência da Informação podem vir a ter. Este estudo, portanto, corrobora com os resultados 
Paulo Roberto Cintra, Ariadne Chloe Furnival, Douglas Henrique Milanez.

Vantagens de citação do acesso aberto em periódicos selecionados da ciência da informação: uma análise ampliada aos indicadores altmétricos

encontrados acerca da OACA para as demais áreas do conhecimento e, além disso, mostra que essas vantagens se mantêm até o oitavo ano após a publicação do artigo.

A pesquisa indicou que o número de publicações disponibilizadas em acesso aberto nos periódicos caracterizados, através do pagamento das taxas de processamento de artigos, foi de, em média, 4\%. Ademais, verificou-se que $52 \%$ das publicações em acesso fechado nas revistas científicas puderam ser encontradas online, em seu texto completo e sem barreiras ao acesso, através de pesquisas em um mecanismo de busca. Logo, nota-se que não são todos os autores que realizam o autoarquivamento de suas publicações após o período de embargo, o que contribui para a manutenção de uma grande parcela da literatura que ainda permanece em acesso fechado, ou seja, sujeita à subscrição dos periódicos.

Uma razão para a ocorrência dessa situação está relacionada ao desinteresse dos próprios pesquisadores em fazer o autoarquivamento de suas publicações, que ainda se mantém distantes do movimento do acesso aberto. Outro motivo para essa situação pode estar relacionado ao baixo número de repositórios institucionais que permitam aos autores realizar o depósito de seus artigos. Dessa forma, incentivos à criação de repositórios mais amplos pelas instituições de ensino e pesquisa, que possibilitem ir além do arquivamento reservado a teses e dissertações, poderão colaborar de forma mais efetiva para a transmissão de conhecimentos dentro da comunidade acadêmica e, assim, contribuir para o alcance de alguns dos objetivos do Acesso Aberto.

Os dados de citação do Google Acadêmico, de modo geral, ratificaram a vantagem oferecida pelo acesso aberto. Ademais, observou-se que o Google Acadêmico conseguiu abranger um maior número de citações recebidas pelos artigos do que aquele aferido na Web of Science. Tal fato mostra que, para a área da Ciência da Informação, a avaliação da produção científica fundamentada exclusivamente na base bibliográfica da empresa Thomson Reuters não reflete com fidedignidade a realidade desse campo científico, sendo necessário estudos de outros métodos para esse tipo de apreciação. 
Paulo Roberto Cintra, Ariadne Chloe Furnival, Douglas Henrique Milanez.

Vantagens de citação do acesso aberto em periódicos selecionados da ciência da informação: uma análise ampliada aos indicadores altmétricos

Outrossim, os artigos disponibilizados imediatamente em acesso aberto pelos autores nos periódicos, através do pagamento dos APCs, apresentaram uma vantagem maior na quantidade de menções na web social, quando comparado com os documentos que foram autoarquivados em repositórios institucionais. Esta observação pode estar associada à exigência dos periódicos acerca de um período de embargo entre a publicação do artigo e o autoarquivamento, o que conduz a uma disponibilização tardia desses documentos à academia científica.

Por fim, embora os dados altmétricos venham sendo discutidos na literatura como medida complementar à análise tradicional bibliométrica, as avaliações de produção científica baseadas prioritariamente no número de citações que um artigo recebe ainda são predominantes. Provavelmente essa predominância seja consequência do desafiador processo que é a obtenção dos indicadores altmétricos. Considerando as diferenças entre os valores médios de citação obtidos pelos periódicos analisados, com relação ao autoarquivamento e a disponibilização imediata em acesso aberto via pagamento de APC, sugere-se que novos estudos investiguem a viabilidade de retorno sobre o investimento despendido para pagamento destas taxas, refletidos no número de citações obtidas nos anos imediatamente posteriores à data da publicação. Além da ampliação deste estudo para os demais periódicos da área da Ciência da Informação, esta análise também poderá ser estendida a outros campos do conhecimento.

\section{AGRADECIMENTOS}

À Fundação de Amparo à Pesquisa do Estado de São Paulo (FAPESP) - processos n. 2015/16872-2 e 2015/18878-8. 
Paulo Roberto Cintra, Ariadne Chloe Furnival, Douglas Henrique Milanez.

Vantagens de citação do acesso aberto em periódicos selecionados da ciência da informação: uma análise ampliada aos indicadores altmétricos

\section{REFERÊNCIAS}

ANTELMAN, K. Do open-access articles have a greater research impact? College \& Research Libraries, v.65, n.5, p.372-382, set. 2004.

ARCHAMBAULT, E. et al. Proportion of open access peer-reviewed papers at the European and world levels - 2004-2011. 2013. Disponível em: <http://www.science-metrix.com/pdf/SM EC OA Availability 2004-2011.pdf> Acesso em: 21 abr. 2016.

ARCHAMBAULT, E. et al. Research impact of Paywalled versus Open Access papers. 2016. Disponível em: <http://www.1science.com/oanumbr.html> Acesso em 29 ago. 2016.

ATCHINSON, A.; BULL, J. Will open access get me cited? an analysis of the efficacy of open access publishing in political science. PS: Political Science \& Politics, Cambridge, v.48, n.1, p.129-137, jan. 2014. Disponível em: <http://dx.doi.org/10.1017/S1049096514001668> Acesso em: 24 jul. 2016.

BOAI. Budapest Open Access Initiative. 2016. Disponível em:

<http://www.budapestopenaccessinitiative.org/read> Acesso em: 09 abr. 2016.

COSTA, M.P. da; LEITE, F.C.L. Open access in the world and Latin America: a review since the Budapest Open Access Initiative. Transinformação, Campinas, v.28, n.1, p.33-46, abr. 2016. Disponível em: $<$ http://dx.doi.org/10.1590/2318-08892016002800003> Acesso em: 01 ago. 2016.

DROESCHER, F.D.; SILVA, E.L. O acesso aberto e o uso da informação científica. Investigación bibliotecológica, México, v.29, n.65, jan./abr. 2015. Disponível em: $<$ http://www.scielo.org.mx/scielo.php?script=sci arttext\&pid=S0187358X2015000100008> Acesso em: 25 jul. 2016.

FRANDSEN, T.F. The effects of open access on un-published documents: a case study of economics working papers. Journal of Informetrics, Hasselt, v.3, n.2, p.124-133, 2009.

GALLIGAN, F.; DYAS-CORREIA, S. Altmetrics: rethinking the way we measure. Serials Review, Philadelphia, v.39, n.1, p. 56-61, mar. 2013. Disponível em: <http://www.sciencedirect.com/science/article/pii/S009879131300004X> Acesso em: 29 abr. 2015.

HARNAD, S. OA impact advantage = EA + (AA) + (QB) + QA + (CA) + UA. School of Electronics \& Computer Science, University of Southampton, 2005. Disponível em: <http://eprints.soton.ac.uk/262085> Acesso em: 21 abr. 2016. 
Paulo Roberto Cintra, Ariadne Chloe Furnival, Douglas Henrique Milanez.

Vantagens de citação do acesso aberto em periódicos selecionados da ciência da informação: uma análise ampliada aos indicadores altmétricos

HARNAD, S.; BRODY, T. Comparing the impact of open access (OA) vs. nonOA articles in the same journals. D-Lib Magazine, v.10, n.6, jun. 2004.

HAUSTEIN, S. et al. Coverage and adoption of altmetrics sources in the bibliometric community. 2013. Disponível em: <http://arxiv.org/ftp/arxiv/papers/1304/1304.7300.pdf> Acesso em: 02 mai. 2015.

KOUSHA, K.; THELWALL, M. The web impact of open access social science research. Library \& Information Science Research, v.29, p.495-507, 2007.

LAWRENCE, S. Free online availability substantially increases a paper's impact. Nature, 2001. Disponível em:

<http://www.nature.com/nature/debates/e-access/Articles/lawrence.html> Acesso em: 21 abr. 2016.

MAFLAHI, N.; THELWALL, M. When are readership counts as useful as citation counts? Scopus versus Mendeley for LIS Journals. Journal of the Association for Information Science and Technology, v.67, n.1, p.191-199, 2016.

MEADOWS, A.J. Comunicação científica. Brasília: Briquet de Lemos Livros, 1999.

OKUBO, Y. Bibliometric indicators and analysis of research systems: methods and examples. Paris: OECD Publishing, 1997.

PINFIELD, S. et al. The "total cost of publication" in a hybrid open-access environment: institutional approaches to funding journal article-processing charges in combination with subscriptions. Journal of the Association for Information Science and Technology, 2015. Disponível em: <http://dx.doi.org/10.1002/asi.23446> Acesso em: 10 maio 2016.

PRIEM, J. et al. Altmetrics: a manifesto. 2010. Disponível em: <http://altmetrics.org/manifesto> Acesso em: 17 jun. 2015.

RESEARCHGATE. About us. 2016. Disponível em: <https://www.researchgate.net/about> Acesso em: 21 abr. 2016.

ROSEN, H.S.; GAYER, T. Public finance. 10ed. New York: McGraw-Hill Global Education Holdings, 2014.

SOUZA, I.V.P. de. Altmetria: métricas alternativas do impacto da comunicação científica. 2014. 104p. Dissertação (Mestrado em Ciência da Informação) Universidade Federal Fluminense, Niterói, 2014.

STIGLITZ, J. Knowledge as a Global Public Good. In: KAUL, I.; GRUNBERG, I.; STERN, M.A. (eds.) Global public goods: international cooperation in the 21st century. New York: Oxford University Press, 1999. p.308-325. 
Paulo Roberto Cintra, Ariadne Chloe Furnival, Douglas Henrique Milanez.

Vantagens de citação do acesso aberto em periódicos selecionados da ciência da informação: uma análise ampliada aos indicadores altmétricos

SWAN, A. The Open Access citation advantage: studies and results to date. Technical Report, School of Electronics \& Computer Science, University of Southampton. 2010. Disponível em: <http://eprints.ecs.soton.ac.uk/18516> Acesso em: 02 mai. 2015.

TARGINO, M.G. Comunicação científica: uma revisão de seus elementos básicos. Informação \& Sociedade: Estudos, Paraíba, v.10, n.2, p. 37-85, 2000.

WANG, $X$. et al. The open access advantage considering citation, article usage and social media attention. Scientometrics, v.103, n.2, p. 555-564, mar. 2015. Disponível em: <http://link.springer.com/article/10.1007\%2Fs11192-015-15470). Acesso em: 25 abr. 2015.

WILLINSKY, J. The access principle: the case for open access to research and scholarship. Cambridge, Massachusetts: MIT Press, 2006.

XIA, J. et al. Multiple open access availability and citation impact. Journal of Information Science, v.37, n.1, p.19-28, fev. 2011.

\title{
Title
}

Open Access Citation Advantage in selected Information Science journals: an extended analysis to altmetrics indicators

\begin{abstract}
Introduction: Open access refers to scientific literature available free of charge and free of copyright restrictions and licensing for its reuse. An increase in the total number of citations received by articles available in open access in relation to those of restricted, pay-walled access is expected, according to the Open Access Citation Advantage hypothesis. Objective: Assess the possible citation advantages and mentions on the social web that open access can offer to the Information Science area.

Methodology: Bibliometric and altmetric indicators were analyzed in two journals: Journal of the American Society for Information Science and Scientometrics. Data collection was conducted in the Web of Science, Google Scholar, Altmetric.com and Mendeley. Results: The results indicated that for both journals, open access offers an advantage in the number of citations received by articles. It was also demonstrated that the advantage is maintained over time. Conclusions: This research confirmed the hypothesis of an Open Access Citation Advantage for the journals analyzed in the area of Information Science. This pattern was also observed for the altmetric data.
\end{abstract}

Keywords: Open Access. Journals. Information Science. Bibliometrics. Altmetrics.

\section{Titulo}

Ventajas de cita del acceso abierto en revistas seleccionadas de la Ciencia de la Información: un análisis extendido a los indicadores altmétricos 
Paulo Roberto Cintra, Ariadne Chloe Furnival, Douglas Henrique Milanez.

Vantagens de citação do acesso aberto em periódicos selecionados da ciência da informação: uma análise ampliada aos indicadores altmétricos

\begin{abstract}
Resumen
Introducción: El acceso abierto se refiere a la literatura científica disponible libremente en Internet, permitiendo a cualquier usuario tener acceso a esta información de forma gratuita y libre de restricciones de derechos de autor y licencias para su reutilización. La hipótesis del Open Access Citation Advantage, se refiere a un aumento esperado en el número total de citas recibidas por los artículos disponibles en acceso abierto en relación a los de acceso restringido. Objetivo: Analizar las posibles ventajas que el acceso abierto puede ofrecer en el área de Ciencias de la Información. Metodología: Se analizaron indicadores bibliométricos y datos altmétricos en dos revistas científicas: Journal of the American Society for Information Science y Scientometrics. Los datos recogidos se realizaron en la Web of Science, Google Scholar, Altmetric.com y Mendeley. Resultados: Los resultados indicaron que el acceso abierto ofrece ventaja en el número de citas recibidas por los artículos en ambos compartimientos. Este patrón también se observó para los datos altmétricos. Conclusiones: La investigación confirmó la hipótesis de Open Access Citation Advantage para las revistas analizadas del campo de la Ciencia de la Información y permitió evaluar que esta ventaja se mantiene a lo largo de los años.
\end{abstract}

Palabras clave: Acceso Abierto. Revistas. Ciencia de la Información. Bibliometría. Altmetría.

Recebido: 12.02.2016

Aceito: 25.03.2017 